\section{Two Decades of Research on the Biosynthesis of Saccharides}

\author{
Luis F. Leloir
}

Our work on the biosynthesis of oligo- and polysaccharides started about 1946, not by a deliberate selection of the subject but because it came to us. Due to the phenomenal progress of biochemistry our initial experiments seem to belong to the paleolithic period but fortunately there are also some very recent and exciting advances in the field.

After returning from Cambridge in 1936 I did some work with J. M. Muñoz on the oxidation of fatty acids in liver. We managed to prepare a cell-free system which was active when suitably supplemented and this was a novel result since the process of oxidation was believed to require the integrity of the cells. I suppose the young generation of biochemists finds it hard to understand many of the things which we believed at that time.

After that came an incursion into the field of renal hypertension with $E$. Braun Menéndez, J. C. Fasciolo, and a. C. Taquini. This work was carried out quite rapidly and was rather successful.

Then I worked at Carl F. Cori's laboratory in St. Louis and with D. E. Green at Columbia University.

On returning to Buenos Aires in 1945

Copyright (1) 1971 by the Nobel Foundation.

The author is director of the Instituto de Investigaciones Bioquímicas, Buenos Aires, Argentina. This article is the lecture he delivered in Stockholm, Sweden, on 11 December 1970 when he received the Nobel Prize in Chemistry. It is published here with the permission of the Nobel Foundation and will also be included in the complete volume of Les Prix Nobel en 1970 as well as in the series Nobel Lectures (in English) published by the Elsevier Publishing Company, Amsterdam and New York.
I started to work with R. Caputto and R. Trucco. Dr. Caputto had done some research on the mammary gland and had the idea that glycogen was transformed into lactose. At the time one had to rely on osazones for identification and we soon reached a dead end. On looking back I think that what we were observing was the degradation of glycogen by amylase.

We then decided to study the breakdown of lactose by a yeast Saccharomyces fragilis with the idea that this would give us information on the mechanism of synthesis. In fact, it did give us information but only after a long and tortuous process.

First we studied the lactase, then the phosphorylation of galactose (1) and the transformation of galactose 1-phosphate. What we measured was the increase in reducing power of the following reaction sequence:

galactose 1-phosphate $\rightarrow$

glucose 1-phosphate $\rightarrow$ glucose 6-phosphate

We soon found that a thermostable factor was required and set out to isolate it in collaboration with C. E. Cardini and A. C. Paladini.

At the time things were not so easy because we did not have the powerful methods which we have nowadays and because we were working under rather poor conditions.

The results of our experiments were very confusing because we did not know that we were dealing with two thermostable factors. Finally we realized what was happening and we concentrated on the purification of the factor involved in the second reaction. That is in the phosphoglucomutase reaction.

We sent a letter to the editors of Archives of Biochemistry (2) describing a new cofactor and mentioned that Kendall and Strickland (3) had previously described an activation by fructose 1,6diphosphate but that our cofactor was different. After we had sent the manuscript we happened to test fructose 1,6-diphosphate again and obtained a strong activation. Furthermore our purified preparations were loaded with fructose 1,6-diphosphate. We had decided to ask that the letter be returned but as a consequence of much worrying we struck on the idea that the activator might be glucose 1,6-diphosphate. Since the latter compound has the reducing group blocked we reasoned that it should be alkali stable. Strangely enough everything turned out as expected. If it had not been for this mistake we might still be talking of the allosteric effect of fructose 1,6-diphosphate on phosphoglucomutase.

When we finished working with glucose 1,6-diphosphate we continued with the other cofactor. The concentrates were found to absorb light at 260 nanometers and had a spectrum similar to that of adenosine but with some differences. At the time the only soluble nucleotides known to be present in tissues were the inosine and adenosine nucleotides. It was an exciting day when Caputto came in one morning with a Journal of Biological Chemistry which showed the absorption spectrum of uridine. It looked identical to that of our cofactor. After measuring the glucose and phosphate content and doing a titration curve, the structure shown in Fig. 1 was proposed (4). This first sugar nucleotide was named uridine diphosphate glucose: UDPG. Its structure was confirmed by synthesis some 5 years later by Todd and his co-workers in Cambridge. The mechanism by which UDP-glucose acts as a cofactor in the galactose 1-phosphate $\rightarrow$ glucose 1-phosphate transformation became understandable when it was found that on in- 


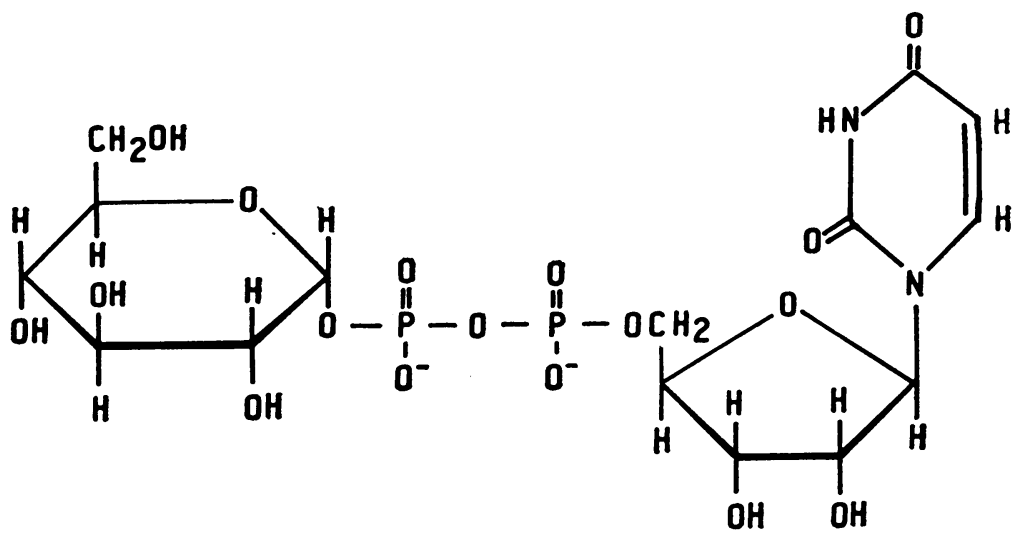

Fig. 1. Uridine diphosphate glucose (UDPG).

cubation with yeast extracts part of the UDP-glucose was transformed into UDP-galactose (5). After this we wrote the equations as follows:

galactose 1-phosphate + UPD-glucose $\leftrightharpoons$ glucose 1-phosphate + UPD-galactose

UDP-galactose $\leq$ UDP-glucose

Sum: galactose 1-phosphate $\leftrightharpoons$ glucose 1-phosphate

We used to call the whole system waldenase but Kalckar (6) suggested the names of uridylyl transferase and 4epimerase for the enzymes corresponding to Eqs. 2 and 3, respectively.

After we found that yeast which was not adapted to galactose contained a lot of UDP-glucose we concluded that UDP-glucose should have some other function besides being a cofactor of galactose metabolism. I don't know if the reasoning was quite right but the facts were. For some years it was a joke in the laboratory because we were always asking: "What's the use of UDPglucose?"

Since we had a method for estimating UDP-glucose with the galactose 1phosphate $\rightarrow$ glucose 6-phosphate reaction, we began to measure the disappearance of UDP-glucose in different extracts and under different conditions. With yeast extracts it was observed that the addition of glucose 6-phosphate increased the disappearance of UPDglucose, and finally this was found to be due to the formation of trehalose phosphate, a substance which had been isolated from yeast many years before by Robison and Morgan (7). The reaction is as follows:

UDP-glucose + glucose 6-phosphate $\rightarrow$ trehalose phosphate + UDP

This work which was carried out with Cabib (8) described the first case in which UDP-glucose was found to act as a glucose donor. Such a role had been suggested by Buchanan et al. (9) and by Kalckar (10).

Once we had found one transfer reaction we were soon able to detect another one using wheat germ extracts. Actually we found two enzymes, one which gave rise to the formation of sucrose (11) and another which gave sucrose phosphate (12) as follows:

UDP-glucose + fructose $\leftrightharpoons$

$$
\text { sucrose + UDP }
$$

UDP-glucose + fructose 6-phosphate $\leftrightharpoons$ sucrose phosphate + UDP

This was a rather interesting finding because it explained the mechanism of sucrose synthesis in plants.

Another novel result of that period was the isolation of UDP- $N$-acetylglucosamine (13). This substance was first detected as an impurity of UDP-glucose concentrates and we used to call it UDP-X until we were able to identify the sugar moiety as $N$-acetylglucosamine. It is now known to be involved in the biosynthesis of bacterial cell walls and mucoproteins.

Other members of the sugar nucleotide family were isolated in our laboratory. In 1954 (14) GDP-mannose was found in yeast extracts, and later Pontis (15) detected UDP- $N$-acetylgalactosamine in liver. These substances are now known to be involved in the biosynthesis of mannan (16) and of some proteoglycans.

Other laboratories made important contributions. The identification of UDP-glucuronic acid as a donor for the formation of glucuronides (17) was the first example of a transfer reaction from a sugar nucleotide.

Another important compound was detected by Park and Johnson (18) at about the same time that we isolated
UDP-glucose. They found that a compound containing uridine accumulated in Staphylococcus treated with penicillin. This substance turned out to be difficult to identify because the sugar moiety was unknown at the time. This compound which kept biochemists in the dark behaved like a strange hexosamine and it was Strange and Dark (19) who first obtained a crystalline preparation. We now know that the sugar moiety is acetylglucosamine joined to lactic acid forming an ether linkage and the substance has been named muramic acid. The isolation of UDPmuramic acid was the starting point of the beautiful work carried out on bacterial cell wall synthesis which owes so much to Park and Strominger.

The number of known sugar nucleotides increased progressively for several years and in the 1963 census (208 they numbered more than 48 . Further more, many enzymes involved in intero conversion reactions have been studicd कि Herman Kalckar's group found that NAD is required in the UDP-glucose 4-epimerase reaction and it is believe重 that the glucose moiety of UDP-glucose is oxidized to a 4-keto intermediate which can then be reduced either t命 glucose or galactose.

Several other more complicated trans $\stackrel{\frac{\Omega}{\Phi}}{\frac{\Phi}{2}}$ formations have been carefully studied for instance, the transformation of GDP-mannose to GDP-fucose whiclo requires a reduction at $\mathrm{C}-6$ and inver sions at -3 and -5 (21). A similar case is the formation of TDP-rhamnose from TDP-glucose in which $\mathrm{OH}$ group at C-3, -5 , and -6 become inverted and a reduction at C-6 occurs (22).

\section{Polysaccharides}

Many transfer reactions from sugar nucleotides have been detected. Thus, Glaser and Brown (23) detected a transfer of $N$-acetylglucosamine from UDP$N$-acetylglucosamine to chitin catalyzed by mold extracts. The formation of a $\beta$ 1,3 glucan (callose) from UDP-glucose and of xylan from UDP-xylose was obtained by incubation with plant extracts (24).

A transfer from UDP-glucose to cellulose was also described by Glaser (25) working with Acetobacter xylinum which is a cellulose-forming bacterium. Later it was found that the donor for cellulose formation in plants is GDPglucose (26).

In our laboratory (27) we were able 
to detect the formation of glycogen from UDP-glucose (Eq. 8) with liver and muscle enzymes:

UDP-glucose $+\mathrm{G}_{n} \rightarrow \mathrm{UDP}+\mathrm{G}_{n+1}$

In this equation $\mathrm{G}_{n}$ represents a glycogen molecule and $G_{n+1}$ the same after addition of a glucosyl residue joined $\alpha-1,4$.

The search for this enzyme glycogen synthetase or transferase was stimulated by reading a book by Herman Niemeyer (28) and its detection was a rather interesting finding since until then the synthesis of glycogen was believed to occur by reversal of the phosphorylase reaction (Eq. 9):

(glucose $)_{n+1}+$ inorganic phosphate $\leftrightharpoons$
$\quad(\text { glucose })_{n}+$ glucose 1-phosphate

The same enzyme was thought to be involved in synthesis and in degradation. Another finding of considerable interest was that glucose 6-phosphate acts as an activator of glycogen synthetase.

Many years before, the Coris had found that muscle phosphorylase has two forms which differ in their requirement for adenylic acid. Similarly, J. Larner and C. Villar-Palasi described two interconvertible forms of glycogen synthetase, one active per se and another which requires glucose 6-phosphate. From then on a lot of work has been done on the regulation of glycogen metabolism.

Both phosphorylase and glycogen synthetase are regulated by the concentration of metabolites (adenylic acid and glucose 6-phosphate, respectively, as well as others, such as ATP) and by reversible conversion of active to inactive forms. The latter changes are brought about by the action of several enzymes on one another. The picture which we have of the mechanism of glycogen regulation is too complicated to be shown here [for reviews see (29)].

Most of the studies on the biosynthesis of polysaccharides have consisted only in measuring the transfer of minute amounts of radioactive sugars. However, the studies should go further and we should be able to obtain in vitro polysaccharides identical to those made by cells. Some work of this type has been done with glycogen. One can obtain glycogen by incubating glucose 1-phosphate with phosphorylase or UDP-glucose with glycogen synthetase (in both cases with branching enzyme). The resulting products have been found to be of high molecular weight but different as judged by their pattern of degrada-

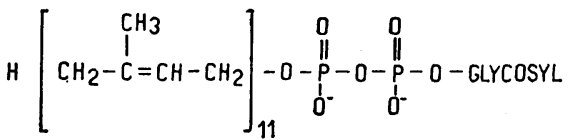

Fig. 2. Antigen carrier lipid.

tion by acid or alkali. The product formed with UDP-glucose and glycogen synthetase proved to be identical to that isolated from liver (30).

A logical extension of our work on glycogen was to investigate the formation of starch in plants. Enzymes were found which catalyzed the transfer of radioactivity from UDP-glucose labeled in the glucose moiety to starch (31). Studies on the specificity of the enzyme using synthetic nucleotides showed that ADP-glucose was used about ten times faster (32). This led to a search for ADP-glucose in natural sources which resulted in its isolation from corn (33). An enzyme which can synthesize ADP. glucose was found by Espada (34).

Since then a lot of work has been done on the subject by several workers, particularly by Carlos Cardini, Rosalía Frydman, Jack Preiss, T. Akazawa, and others.

In Euglena the reserve polysaccharide is a $\beta-1,3$ linked glucan usually called paramylon. Its synthesis was studied by Goldemberg and Marechal (35) who found that it is formed from UDPglucose.

Many more transfer reactions have been described so that the search was becoming monotonous.

\section{Lipid Intermediates}

From the data reported it may be concluded that most of the di-, oligo-, and polysaccharides which occur in nature in an amazing variety are synthesized from nucleotide sugars. However, at least in some cases, it seems that the transfer is not direct but is mediated by lipid intermediates. This has been one of the most important findings of the last years and it is linked to the work of several groups (Osborn, Horecker, Strominger, Robbins, Lennartz, and others). The structure of the first lipid intermediate detected in bacteria (36) is shown in Fig. 2.

The structure of the compound was established in very small amounts, mainly by mass spectroscopy. The compound, undecaprenol pyrophosphate, contains 11 isoprene residues, one of them bearing an $\mathrm{OH}$ group joined to a pyrophosphate which in turn is linked to sugar residues.

The role of the carrier lipid in the formation of Salmonella lipopolysaccharide may be summarized in the following equations (where LP stands for the monophosphorylated lipid intermediate)

$$
\begin{aligned}
& \text { LP + UDP-galactose } \rightarrow \\
& \text { LPP-galactose + UMP }
\end{aligned}
$$

LPP-galactose + TDP-rhamnose $\rightarrow$

LPP-galactose-rhamnose + UDP

LPP-galactose-rhamnose + GDP-mannose $\rightarrow$

LPP-galactose-rhamnose-mannose + GDP

$n$ LPP galactose-rhamnose-mannose $\rightarrow$ LPP(galactose-rhamnose-mannose $)_{n}+$ $(n-1)$ LPP

LPP(galactose-rhamnose-mannose $)_{n}+$ core $\rightarrow$

(galactose-rhamnose-mannose $)_{n} \cdot$ core + LPP (14)

$$
\mathrm{LPP} \rightarrow \mathrm{LP}+\mathrm{P}
$$

In the first step (Eq. 10) there is a transfer of galactose 1-phosphate so that the lipid pyrophosphate and UMP are formed. Then rhamnose and mannose are added from the respective sugar nucleotides. Finally the trisaccharide units are transferred so as to form long chains ( $n=$ about 60 ) of galactose-rhamnose-mannose repeating units joined to the intermediate. In the next step (Eq. 14) these would be transferred to the core of the lipopolysaccharide.

Undecaprenol pyrophosphate plays a similar role in the formation of bacterial cell walls in staphylococci. The wall material, murein, is formed by alternating units of acetylglucosamine and muramic acid residues. These chains are cross-linked by peptides joined to the muramic acid residues.

The mechanism by which the cell wall is assembled has been elucidated mainly by the work of Strominger's group (37) and can be written as follows (M $=N$-acetyl muramic acid joined to the following peptide: L-ala D-glu L-lys D-ala D-ala; $N$-Ac stands for $N$-acetylglucosamine):

$$
\begin{gathered}
\text { UDPM + LP } \rightarrow \text { LPPM + UMP } \\
\text { UDP } N-\text { Ac }+ \text { LPPM } \rightarrow \\
\text { LPPM } N \text {-Ac + UDP } \\
\text { tRNA gly + LPPM } N \text {-Ac } \rightarrow \\
\text { tRNA + LPPM } N \text {-Ac gly } \\
\text { LPPM } N \text {-Ac gly + acceptor } \rightarrow \\
(\text { M- } N \text {-Ac gly)-acceptor + LPP }
\end{gathered}
$$


The first step (Eq. 16) is a transfer of muramyl peptide phosphate from the corresponding uridine nucleotide (one of the compounds isolated by Park) to undecaprenol monophosphate. Next (Eq. 17) $N$-acetylglucosamine is transferred from UDP- $N$-acetylglucosamine. After that (Eq. 18) one more amino acid is added (from a transfer ribonucleic acid) and then the whole disaccharide peptide is added to a part of the growing cell wall (referred to as acceptor in Eq. 19). After this the cross-links are established between the peptide chains and the cell wall is complete.

Another piece of work dealing with lipid intermediates should be mentioned. This refers to the formation of mannan by Micrococcus lysodeikticus (38). The reactions are as follows:

GDP-mannose + undecaprenol-P $\rightarrow$

GDP + undecaprenol-P-mannose

undecaprenol-P-mannose + acceptor $\rightarrow$ mannose-acceptor + undecaprenol-P

The difference with the previously mentioned cases is that in the first reaction (Eq. 20) the sugar without the phosphate is transferred so that no pyrophosphate is formed.

While all this work was going on, Dankert, who had been working with Robbin's group, returned to Buenos Aires and transmitted to us his enthusiasm for polyprenols.

\section{A Polyprenol Intermediate in}

\section{Animal Tissues}

A group working at the University of Liverpool formed by Morton, Hemming, and others has studied carefully the different polyprenols found in nature. The general formula is as follows:

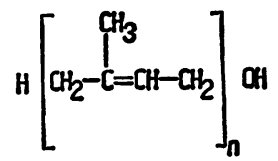

Many different types of compounds were detected which differ in the number $n$ of isoprene residues, in the amount of cis or trans double bonds, and also in that some of the double bonds may be saturated.

The compound isolated from animal tissues was named dolichol. In this substance the number of isoprene units is around 20 (it can vary from 16 to 23), and two of the double bonds are trans. Furthermore, the double bond nearest to the alcohol group is saturated. Many other compounds were isolated from different sources (39).

With N. Behrens (40) we have studied a process occurring in liver in which it turned out that a phosphate of dolichol is involved. The reactions may be written as follows:

UDP-glucose + DMP $\rightarrow$
DMP-glucose +UDP
DMP-glucose $+\mathrm{E} \rightarrow$ glucose-E + DMP

(23)

$$
\text { glucose- } \mathrm{E} \rightarrow \text { glucose }+\mathrm{E}
$$

In these equations DMP stands for dolichol monophosphate and $\mathrm{E}$ for an endogenous acceptor believed to be a protein.

The studies were carried out by incubating liver microsomes with radioactive UDP-glucose. It was found that a product soluble in organic solvents was formed. Further work showed that the reaction shown in Eq. 22 could be carried out so as to measure the lipid acceptor (DMP in Eq. 22). This allowed a purification process to be developed. The concentrates obtained gave infrared spectra having similarities with polyprenols. The compound had acidic character and was relatively stable to acid and alkali. It differed from undecaprenol phosphate in that the latter is acid labile. It was reasoned that this difference could be due to the fact that in undecaprenol there is a double bond near the phosphate which is not present in dolichol. With this idea in mind the identification of our lipid acceptor was approached from another angle. Dolichol was prepared from liver (41), phosphorylated chemically, and then tested for activity as lipid acceptor. The synthetic compound turned out to be identical, in all the properties tested, to that obtained from natural sources. For this reason we refer to it as dolichol monophosphate.

As to the glucosylated compound (DMP-glucose) it was found to be very labile to acid and to be decomposed by alkali, giving 1,6-anhydroglucosan. The following reaction (Eq. 23) could be studied independently from the first by using DMP-glucose prepared in a preliminary run. The optimal conditions for activity were determined. This step (Eq. 23) does not require any cation in contrast to the reaction shown in Eq. 22 in which $\mathrm{Mg}^{2}+$ ions are necessary. Detergents are required in both steps.

The product formed from DMP. glucose, indicated as glucose-E in Eq. 23, appears to be a glucosylated protein but work has just started on this point.
There are very few proteins that contain glucose. One of them is collagen which contains glucosyl, galactosyl hydroxy-lysine residues. However the compound formed with liver microsomes seems to be clearly different from collagen. The last reaction (Eq. 24) has not been studied in any detail and could be brought about by some of the glucosidases known to be present in liver.

The possibility that the glucosylation of ceramide, which is the first step in the formation of gangliosides, might be mediated by DMP-glucose has been investigated with results that are not quite conclusive but indicate that DMP. glucose is not involved.

Other sugar nucleotides have been tested and it was found that UDP-Nacetylglucosamine and GDP-mannose can serve as donors for the formation of the corresponding DMP-sugars. Other compounds such as UDP- $N$-acetylgalactosamine and UDP-galactose gave negative results (42).

The study of the lipid intermediates is becoming most interesting. The va- $\overline{0}$ riety of polyprenols is large since they may vary in chain length, number of cis or trans double bonds, and degree of saturation. Furthermore they may have one or two phosphates and carry different sugars. The variety of polyprenol phosphate sugars may turn out to be as large as that of sugar nucleotides. It has been suggested that their role may beĉ̣ to provide a lipophylic moiety to sugars so as to allow them to permeate the lipid layer of membranes. Since in $\frac{\vec{D}}{2}$ Salmonella polyprenol phosphates are involved in the formation of specific $\underset{\omega}{N}$ antigen it seems likely that in animal N tissues they may be responsible for the formation of the surface carbohydrates which are so important in the behavior of contacting cells. These external specific substances and interactions, which Kalckar (43), in one of his penetrating essays, calls "ektobiological," appear to be of great importance in the "social" behavior of cells. Undoubtedly this may become a fascinating problem for future research. Fortunately even after two decades our field of investigation has not become dull or too fashionable.

\section{Acknowledgements}

My whole research career has been influenced by one person, Professor Bernardo A. Houssay, who directed my doctoral thesis and who, during all these years, generously gave me his in- 
valuable advice and friendship. I also owe very much to my friends, colleagues, and co-workers, the names of whom are mentioned in the text.

The help of the "Fundación Campomar," Consejo Nacional de Investigaciones Científicas y Técnicas, Facultad de Ciencias, Exactas y Naturales-Universidad de Buenos Aires, the National Institutes of Health (United States), and the Rockefeller Foundation, which allowed us to carry out our work, is gratefully acknowledged.

\section{References}

1. R. E. Trucco, R. Caputto, L. F. Leloir, N. Mittelman, Arch. Biochem. 18, 137 (1948).

2. R. Caputto, L. F. Lelrir, R. E. Trucco, C. E. Cardini, A. C. Paladini, ibid., p. 201.

3. L. P. Kendall and L. H. Strickland, Biochem. J. 32, 572 (1938).

4. C. E. Cardini, A. C. Paladini, R. Caputto, L. F. Leloir, Nature 165, 191 (1954); R. Caputto, L. F. Leloir, C. E. Cardini, A. C. Paladini, J. Biol. Chem. 184, 333 (1950).

5. L. F. Leloir, Arch. Biochem. Biophys. 33, 186 (1951).

6. H. M. Kalckar, Advan. Enzymol. 20, 111 (1958).

7. R. Rcbison and W. T. Morgan, Biochem. J. 24, 119 (1930).

8. L. F. Leloir and E. Cabib, J. Amer. Chem. Soc. 75, 5445 (1953).

9. J. G. Buchanan, J. A. Bassham, A. A. Benscn, D. F. Bradley, M. Calvin, L. L. Daus, M. Goodman, P. Hayes, V. H. Lynch, L. T.
Norris, A. T. Wilson, Phosphorus Metabolism (Johns Hopkins Press, Baltimore, 1952), vol. 2, p. 440 .

10. H. M. Kalckar, The Mechanism of Enzyme Action (Johns Hopkins Press, Baltimore, 1954), p. 675.

11. C. E. Cardini, L. F. Leloir, J. Chiriboga, J. Biol. Chem. 214, 149 (1955).

12. L. F. Leloir and C. E. Cardini, ibid., p. 157.

13. E. Cabib, L. F. Leloir, C. E. Cardini, ibid. 203, 1055 (1953).

14. E. Cabib and L. F. Leloir, ibid. 206, 779 (1954).

15. H. G. Pontis, ibid. 216, 195 (1955).

16. N. H. Behrens and E. Cabib, ibid. 243, 502 (1968).

17. G. J. Dutton and I. D. E. Storey, Biochem. J. 53, xxxvii (1953).

18. J. T. Park and M. J. Johnson, J. Biol. Chem. 179, 585 (1949); J. T. Park, ibid. 194, 887, 885,897 (1952).

19. R. E. Strange and F. A. Dark, Nature 177, 186 (1956).

20. E. Cabib, Annu. Rev. Biochem. 32, 321 (1963).

21. V. Ginsburg, J. Amer. Chem. Soc. 80, 4426 (1958).

22. L. Glaser and S. Kornfeld, J. Biol. Chem. 236, 1795 (1961); J. H. Pazur and E. W. Shuey, ibid., p. 1780 .

23. L. Glaser and D. H. Brown, Biochim. Biophys. Acta 23, 449 (1957).

24. D. S. Feingold, E. F. Neufeld, W. Z. Hassid, J. Biol. Chem. 233, 783 (1958); ibid. 234, 488 (1959).

25. L. Glaser, Biochim. Biophys. Acta 25, 436 (1957).

26. A. D. Elbein, G. A. Barber, W. Z. Hassid, J. Amer. Chem. Soc. 86, 309 (1964).

27. L. F. Leloir and C. E. Cardini, ibid. 79 , 6340 (1957); L. F. Leloir, J. M. Olavarría,
S. H. Goldemberg, H. Carminatti, Arch. Biochem. Biophys. 81, 508 (1959).

28. H. Niemeyer, Metabolismo de los Hidratos de Carbono (Universidad de Chile, Santiago, 1955), p. 150.
29. L. F. Leloir, Proc. Pan-Amer. Congr. Endocrinol., Mexico City, 10-15, October 1965 (1966), p. 65; Nat. Cancer Inst. Monogr. No. 27 (1966), p. 3; C. Villar-Palasi and J. Larner, Vitamins Hormones 26, 65 (1968); D. Stetten and M. R. Stetten, Physiol. Rev. 40, 505 (1960); E. W. Sutherland, I. Øye, R. W. Butcher, Recent Progr. Hormone Res. 21, 623 (1965); E. G. Krebs and E. H. Fischer, Vitamins Hormones 22, 399 (1964).

30. J. Mordoh, L. F. Leloir, C. R. Krisman, Proc. Nat. Acad. Sci. U.S. 53, 86 (1965); J. Mordoh, C. R. Krisman, L. F. Leloir, Arch. Biochem. Biophys. 113, 265 (1966); A. J. Parodi, C. R. Krisman, L. F. Leloir, J. Mordoh, ibid. 121, 769 (1967); A. J. Parodi, J. Mordoh, C. R. Krisman, L. F. Leloir, ibid. 132, 111 (1969).

31. M. A. R. de Fekete, L. F. Leloir, C. E. Cardini, Nature 187, 918 (1960); L. F Ieloir, M. A. R. de Fekete, C. E. Cardini, J. Biol. Chem. 236, 636 (1961).

32. E. Recondo and L. F. Leloir, Biochem. Biophys. Res. Commun. 6, 85 (1961).

33. E. Recondo, M. Dankert, L. F. Leloir, ibid. 12, 204 (1963).

34. J. Espada, J. Biol. Chem. 237, 3577 (1962)

35. S. H. Goldemberg and L. R. Marechal, Biochim. Binphys. Acta 71, 743 (1953).

36. A. Wright, M. Dankert, P. Fennesey, P W. Robbins, Proc. Nat. Acad. Sci. U.S. 57, 1798 (1967).

37. Y. Higashi, J. L. Strominger, C. C. Sweeley, ibid., p. 1878 .

38. M. Scher, W. J. Lennartz, C. C. Sweeley, ibid. 59, 1313 (1968).

39. F. W. Hemming, Biochem. J. 113, 23P (1969).

40. N. H. Behrens and L. F. Leloir, Proc. Nat Acad. Sci. U.S. 66, 153 (1970).

41. J. Burgos, F. W. Hemming, J. F. Pennok, R. A. Morton, Biochem. J. 88, 470 (1953).

42. N. H. Behrens, A. J. Parodi, L. F. Leloir, C. R. Krisman, Arch. Biochem. Biophys., in press.

43. H. M. Kalckar, Science 150, 305 (1965).

\section{Ecology of Anthrax}

\author{
Anthrax undergoes a propagation phase in soil \\ before it infects livestock.
}

Glenn B. Van Ness

Anthrax, an infectious disease of herbivorous animals, has caused epidemics in livestock and in man since antiquity. In the acute form, the disease brings about little change in the tissues of dead animals except dark blood and swollen spleens. In man, a malignant carbuncle is suggestive of anthrax. Pulmonary anthrax, or "wool sorter's disease," is a serious and often fatal infection, resulting from the handling of contaminated hair and wool. Anthrax was

Dr. Van Ness is assistant senior staff veterinarian, Technical Services, Animal Health Division, Agricultural Research Service, U.S. Department of Agriculture, Beltsville, Maryland 20705. linked with endemic soil environments long before Bacillus anthracis was identified as the causative organism. Livestock infections in new areas can be traced to areas of the world in which the disease is endemic in the soil. Spores of $B$. anthracis may contaminate bone meal, wool, hair, skins, and animal feeds-all products in world commerce. Outbreaks result from ingestion of or contact with these spores, but anthrax becomes a recurrent problem only when an animal-soil-animal cycle is established. The disease probably came to the New World with European colonists (1). We must know where the disease is likely to appear and where it is established in the soil before we can hope to control it.

Controlling an outbreak of anthrax may include vaccinating and treating the exposed herd. State laws and meat inspection regulations often influence the choice of programs. Since additional outbreaks may follow the first appearance of the disease, it is important to know where these are likely to occur. Livestock affected with anthrax must not be moved, but area quarantines have to be restricted, in order to avoid serious disturbances in the agricultural economy. If we understand the ecology of anthrax, we may be able to institute better regulatory programs.

The discovery (2) that anthrax organisms form spores was one of the significant events in the science of microbiology and disease. Discovery of the spore led to some erroneous and even pernicious conclusions. The spores will survive indefinitely in a dry environment, such as in dust, on string and swabs in a laboratory, and blood spots on clothing. From this observation, anthrax specialists advanced the concept that, once premises are contaminated with anthrax spores, the spores would persist and infect other animals. 


\section{Science}

\section{Two Decades of Research on Biosynthesis of Saccharides}

Luis F. Leloir

Science 172 (3990), 1299-1303.

DOI: 10.1126/science.172.3990.1299

ARTICLE TOOLS

REFERENCES

PERMISSIONS http://science.sciencemag.org/content/172/3990/1299

This article cites 54 articles, 26 of which you can access for free http://science.sciencemag.org/content/172/3990/1299\#BIBL

http://www.sciencemag.org/help/reprints-and-permissions Advancement of Science, 1200 New York Avenue NW, Washington, DC 20005. The title Science is a registered trademark of AAAS.

Copyright (C) 1971 The Authors, some rights reserved; exclusive licensee American Association for the Advancement of Science. No claim to original U.S. Government Works. 